\title{
Front Matter: Volume 7919
}

, "Front Matter: Volume 7919," Proc. SPIE 7919, Vertical External Cavity Surface Emitting Lasers (VECSELs), 791901 (1 April 2011); doi: 10.1117/12.890188

SPIE. Event: SPIE LASE, 2011, San Francisco, California, United States 


\section{PROCEEDINGS OF SPIE}

\section{Vertical External Cavity Surface Emitting Lasers (VECSELS)}

Ursula Keller

Editor

24-25 January 2011

San Francisco, California, United States

Sponsored and Published by

SPIE 
The papers included in this volume were part of the technical conference cited on the cover and title page. Papers were selected and subject to review by the editors and conference program committee. Some conference presentations may not be available for publication. The papers published in these proceedings reflect the work and thoughts of the authors and are published herein as submitted. The publisher is not responsible for the validity of the information or for any outcomes resulting from reliance thereon.

Please use the following format to cite material from this book:

Author(s), "Title of Paper," in Vertical External Cavity Surface Emitting Lasers (VECSELs), edited by Ursula Keller, Proceedings of SPIE Vol. 7919 (SPIE, Bellingham, WA, 2011) Article CID Number.

ISSN 0277-786X

ISBN 9780819484567

Published by

SPIE

P.O. Box 10, Bellingham, Washington 98227-0010 USA

Telephone +1 3606763290 (Pacific Time) · Fax +1 3606471445

SPIE.org

\section{Copyright (C) 2011, Society of Photo-Optical Instrumentation Engineers}

Copying of material in this book for internal or personal use, or for the internal or personal use of specific clients, beyond the fair use provisions granted by the U.S. Copyright Law is authorized by SPIE subject to payment of copying fees. The Transactional Reporting Service base fee for this volume is $\$ 18.00$ per article (or portion thereof), which should be paid directly to the Copyright Clearance Center (CCC), 222 Rosewood Drive, Danvers, MA 01923. Payment may also be made electronically through CCC Online at copyright.com. Other copying for republication, resale, advertising or promotion, or any form of systematic or multiple reproduction of any material in this book is prohibited except with permission in writing from the publisher. The CCC fee code is 0277-786X/11/ \$18.00.

Printed in the United States of America.

Publication of record for individual papers is online in the SPIE Digital Library.

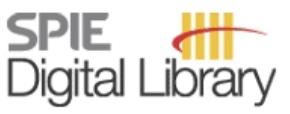

SPIEDigitalLibrary.org

Paper Numbering: Proceedings of SPIE follow an e-First publication model, with papers published first online and then in print and on CD-ROM. Papers are published as they are submitted and meet publication criteria. A unique, consistent, permanent citation identifier (CID) number is assigned to each article at the time of the first publication. Utilization of CIDs allows articles to be fully citable as soon as they are published online, and connects the same identifier to all online, print, and electronic versions of the publication. SPIE uses a six-digit CID article numbering system in which:

- The first four digits correspond to the SPIE volume number.

- The last two digits indicate publication order within the volume using a Base 36 numbering system employing both numerals and letters. These two-number sets start with $00,01,02,03,04$, $05,06,07,08,09,0 A, 0 B \ldots$ OZ, followed by 10-1Z, 20-2Z, etc.

The CID number appears on each page of the manuscript. The complete citation is used on the first page, and an abbreviated version on subsequent pages. Numbers in the index correspond to the last two digits of the six-digit CID number. 


\title{
Contents
}

\author{
vii Conference Committee \\ ix Microfabrication by optical tweezers [7921 02] \\ R. Ghadiri, T. Weigel, C. Esen, A. Ostendorf, Ruhr-Univ. Bochum (Germany)
}

\section{SESSION 1 CW VECSELS: POWER SCALING I}

791903 High power optically pumped VECSELs emitting in 1310-nm and 1550-nm bands (Invited Paper) [7919-02]

A. Sirbu, A. Mereuta, A. Caliman, N. Volet, Q. Zhu, V. lakovlev, Ecole Polytechnique Fédérale de Lausanne (Switzerland); J. Rautiainen, J. Lyytikäinen, O. Okhotnikov, Tampere Univ. of Technology (Finland); J. Walczak, M. Wasiak, T. Czyszanowski, Technical Univ. of Lodz (Poland); E. Kapon, Ecole Polytechnique Fédérale de Lausanne (Switzerland)

$791904 \quad$ High-power $1.25 \mu \mathrm{m}$ InAs QD VECSEL based on resonant periodic gain structure [7919-03] A. R. Albrecht, T. J. Rotter, C. P. Hains, A. Stintz, Ctr. for High Technology Materials, The Univ. of New Mexico (United States); G. Xin, Shanghai Institute of Optics and Fine Mechanics (China); T.-L. Wang, Y. Kaneda, J. V. Moloney, College of Optical Sciences, The Univ. of Arizona (United States); K. J. Malloy, G. Balakrishnan, Ctr. for High Technology Materials, The Univ. of New Mexico (United States)

$79190511 \mathrm{~W}$ single gain element dilute nitride disk laser emitting at $1180 \mathrm{~nm}$ [7919-04] T. Leinonen, V.-M. Korpijärvi, J. Puustinen, Tampere Univ. of Technology (Finland); R. J. Epstein, Areté Associates (United States); M. Guina, Tampere Univ. of Technology (Finland)

791906 Gain coupling VECSELs [7919-05]

R. G. Bedford, Air Force Research Lab. (United States); C. Hessenius, College of Optical Sciences, The Univ. of Arizona (United States); N. Terry, United States Air Force Academy (United States); J. Moloney, M. Fallahi, College of Optical Sciences, The Univ. of Arizona (United States)

\section{SESSION 2 CW VECSELS: POWER SCALING II}

791909 Lateral lasing and ASE reduction in VECSELs [7919-08]

C. Hessenius, M. Fallahi, J. Moloney, College of Optical Sciences, The Univ. of Arizona (United States); R. Bedford, Air Force Research Lab. (United States)

7919 OA $2 \mathbf{~ W ~ c w ~ O P O ~ i n ~ m i d - I R ~ p u m p e d ~ b y ~ O P S L ~ l a s e r ~ i n t r a - c a v i t y ~ r a d i a t i o n ~ [ 7 9 1 9 - 0 9 ] ~}$

A. Caprara, Coherent, Inc. (United States) 
7919 OB Wavelength tunable red AIGalnP-VECSEL emitting at around $660 \mathrm{~nm}$ [7919-10]

T. Schwarzbäck, H. Kahle, M. Eichfelder, W.-M. Schulz, R. Roßbach, M. Jetter, P. Michler, Univ. of Stuttgart (Germany)

7919 OC Blue light source based on spectrally stabilized external dual grating reflector coupled surface emitter array [7919-11]

Y. O. Yilmaz, O. V. Smolski, P. Srinivasan, V. O. Smolski, E. G. Johnson, The Univ. of North Carolina at Charlotte (United States)

7919 OD Recent advances in VECSELs for laser projection applications (Invited Paper) [7919-13]

H. Lindberg, S. Illek, I. Pietzonka, M. Furitsch, A. Plößl, S. Haupt, M. Kühnelt, R. Schulz,

U. Steegmüller, T. Höfer, U. StrauB, OSRAM Opto Semiconductors GmbH (Germany)

\section{SESSION 4 POWER SCALING VECSELS: GROWTH, FABRICATION, AND ELECTRICAL PUMPING}

7919 OF MBE growth challenges of quantum dot saturable absorbers integrated into a MIXSEL [7919-15]

M. Golling, Y. Barbarin, T. Südmeyer, U. Keller, ETH Zurich (Switzerland)

7919 OG Lattice mismatched growth for mid-IR VECSELs (Invited Paper) [7919-16]

G. Balakrishnan, T. J. Rotter, P. Ahirwar, S. P. Clark, V. Patel, A. Albrecht, C. P. Hains, Ctr. for High Technology Materials, The Univ. of New Mexico (United States); Y.-Y. Lai, T. L. Wang, J. M. Yarborough, D. Mathine, Y. Kaneda, J. V. Moloney, College of Optical Sciences, The Univ. of Arizona (United States); J. Hader, Nonlinear Control Strategies Inc. (United States); S. W. Koch, Philipps Univ. of Marburg (Germany)

$7919 \mathrm{OH} \quad$ Recent advances in electrically pumped VECSELs for modelocking (Invited Paper) [7919-17]

Y. Barbarin, ETH Zurich (Switzerland)

7919 Ol Beam quality optimization of electrically pumped VECSELs for passive modelocking [7919-18]

W. P. Pallmann, M. Hoffmann, ETH Zurich (Switzerland); M. Miller, Philips Technologie GmbH U-L-M Photonics (Germany); J. Baier, H. Moench, Philips Research Labs. (Germany); I. Dahhan, B. Witzigmann, Univ. Kassel (Germany); M. Golling, Y. Barbarin, T. Südmeyer, U. Keller, ETH Zurich (Switzerland)

7919 0J High power Bessel beams from EP-VECSELs [7919-19]

G. S. Sokolovskii, S. A. Zolotovskaya, Univ. of Dundee (United Kingdom); S. N. Losev, V. V. Dudelev, A. G. Deryagin, V. I. Kuchinskii, loffe Physico-Technical Institute (Russian Federation); W. Sibbett, Univ. of St. Andrews (United Kingdom); E. U. Rafailov, Univ. of Dundee (United Kingdom)

7919 OK Design and characterization of electrically pumped vertical external cavity surface emitting lasers [7919-20]

J. R. Orchard, D. T. D. Childs, L. C. Lin, B. J. Stevens, D. M. Williams, R. A. Hogg, The Univ. of Sheffield (United Kingdom) 
7919 OL Optimizing electrically pumped vertical extended cavity surface emitting semiconductor lasers (E-VECSELs) (Invited Paper) [7919-21]

J. G. Mclnerney, Univ. College Cork (Ireland) and College of Optical Sciences, The Univ. of Arizona (United States); A. Mooradian, Consultant (United States)

\section{SESSION 5 VECSELS: THEORY AND SIMULATIONS}

7919 OM Design and optimisation of VECSELs for the IR and mid-IR (Invited Paper) [7919-22] J. Hader, College of Optical Sciences, The Univ. of Arizona (United States) and Nonlinear Control Strategies Inc. (United States); T.-L. Wang, J. M. Yarborough, C. A. Dineen, Y. Kaneda, College of Optical Sciences, The Univ. of Arizona (United States); J. V. Moloney, College of Optical Sciences, The Univ. of Arizona (United States) and Nonlinear Control Strategies Inc. (United States); B. Kunert, W. Stolz, Philipps Univ. Marburg (Germany) and NasP-III/V GmbH (Germany); S. W. Koch, Philipps-Univ. Marburg (Germany)

7919 ON Quantum design and nonequilibrium effects in VECSELs (Invited Paper) [7919-23]

A. Bäumner, S. W. Koch, Philipps-Univ. Marburg (Germany)

791900 Design and simulation of electrically pumped mode-locked VECSELs (Invited Paper) [7919-24]

B. Witzigmann, I. Dahhan, Univ. of Kassel (Germany); P. Kreuter, Y. Barbarin, M. Hofmann, W. Pallmann, M. Golling, T. Suedmeyer, U. Keller, ETH Zurich (Switzerland)

7919 OP Scaling high-power ultrafast VECSELs into the femtosecond regime [7919-25]

O. D. Sieber, M. Hoffmann, V. J. Wittwer, W. P. Pallmann, M. Golling, Y. Barbarin, T. Südmeyer, U. Keller, ETH Zurich (Switzerland)

$79190 \mathrm{~N} \quad$ Numerical modelling of optical Stark effect saturable absorbers in mode-locked femtosecond VECSELs [7919-26]

A. H. Quarterman, G. J. Daniell, S. Carswell, K. G. Wilcox, Z. Mihoubi, A. L. Chung,

V. Apostolopoulos, A. C. Tropper, Univ. of Southampton (United Kingdom)

\section{SESSION 6 ULTRAFAST VECSELS AND MIXSELS}

7919 OR Power scaling of the MIXSEL: an integrated picosecond semiconductor laser with $>6$ W average power (Invited Paper) [7919-27]

T. Südmeyer, ETH Zurich (Switzerland)

7919 OS Power scaling of cW and pulsed IR and mid-IR OPSLs (Invited Paper) [7919-28] J. V. Moloney, J. Hader, Nonlinear Control Strategies Inc. (United States) and College of Optical Sciences, The Univ. of Arizona (United States); T.-L. Wang, Y. Ying, Y. Kaneda, J. M. Yarborough, College of Optical Sciences, The Univ. of Arizona (United States); T. J. Rotter, G. Balakrishnan, C. Hains, Ctr. for High Technology Materials (United States) and The Univ. of New Mexico (United States); S. W. Koch, W. Stolz, B. Kunert, Philipps-Univ. Marburg (Germany); R. Bedford, Air Force Research Lab. (United States)

7919 OT QD-based saturable absorbers for ultrafast lasers (Invited Paper) [7919-29]

E. U. Rafailov, S. A. Zolotovskaya, M. Butkus, Univ. of Dundee (United Kingdom) 


\section{SESSION 7 ULTRAFAST VECSELS}

7919 OU Tailoring the wavelength of semiconductor disk lasers (Invited Paper) [7919-30]

O. G. Okhotnikov, Tampere Univ. of Technology (Finland)

7919 OW Timing jitter characterization of a quantum dot SESAM modelocked VECSEL [7919-32]

V. J. Wittwer, W. P. Pallmann, A. E. H. Oehler, B. Rudin, M. Golling, Y. Barbarin, T. Südmeyer,

U. Keller, ETH Zurich (Switzerland)

7919 OX All quantum dot based femtosecond VECSEL [7919-33]

M. Hoffmann, O. D. Sieber, W. P. Pallmann, V. J. Wittwer, ETH Zurich (Switzerland);

I. L. Krestnikov, S. S. Mikhrin, D. A. Livshits, Innolume GmbH (Germany); G. Malcolm,

C. Hamilton, M Squared Lasers Ltd. (United Kingdom); Y. Barbarin, T. Südmeyer, U. Keller,

ETH Zurich (Switzerland)

SESSION $8 \quad$ FEMTOSECOND VECSELS AND NOVEL APPLICATIONS

7919 OY Femtosecond surface emitting lasers (Invited Paper) [7919-34]

A. C. Tropper, Univ. of Southampton (United Kingdom)

$791910 \quad 169 \mathrm{GHz}$ repetition rate passively harmonically mode-locked VECSEL emitting 265 fs pulses [7919-36]

A. H. Quarterman, K. G. Wilcox, A. Perevedentsev, V. Apostolopoulos, Z. Mihoubi,

A. L. Chung, Univ. of Southampton (United Kingdom); H. E. Beere, I. Farrer, D. A. Ritchie, Univ.

of Cambridge (United Kingdom); A. C. Tropper, Univ. of Southampton (United Kingdom)

791912 High peak power femtosecond pulse VECSELs for terahertz time domain spectroscopy [7919-38]

K. G. Wilcox, A. Chung, A. H. Quarterman, Z. Mihoubi, Univ. of Southampton

(United Kingdom); I. Farrer, H. E. Beere, D. A. Ritchie, Univ. of Cambridge (United Kingdom);

V. Apostolopoulos, A. C. Tropper, Univ. of Southampton (United Kingdom)

791913 High-power optically pumped semiconductor laser applications [7919-39]

S. B. Morioka, Coherent, Inc. (United States)

791914 Design and properties of high-power highly coherent single-frequency VECSEL emitting in the near- to mid-IR for photonic applications (Invited Paper) [7919-12]

A. Garnache, A. Laurain, M. Myara, J.-P. Perez, L. Cerutti, Institut d'Electronique du Sud, CNRS, Univ. Montpellier 2 (France); A. Michon, G. Beaudoin, I. Sagnes, Lab. de Photonique et Nanostructures, CNRS (France); P. Cermak, D. Romanini, Lab. de Spectrométrie Physique, CNRS, Univ. Joseph Fourier (France)

Author Index 


\title{
Conference Committee
}

\author{
Symposium Chairs \\ Friedhelm Dorsch, TRUMPF GmbH \& Company KG (Germany) \\ Alberto Piqué, U.S. Naval Research Laboratory (United States) \\ Symposium Cochairs
}

Donald J. Harter, IMRA America, Inc. (United States)

Peter R. Herman, University of Toronto (Canada)

Program Track Chair

Claus P. Streubel, OSRAM GmbH (Germany)

Conference Chair

Ursula Keller, ETH Zurich (Switzerland)

Program Committee

Martin D. Dawson, University of Strathclyde (United Kingdom)

Norman Hodgson, Coherent, Inc. (United States)

Eli Elyahou Kapon, Ecole Polytechnique Fédérale de Lausanne (Switzerland)

Jerome V. Moloney, College of Optical Sciences, The University of Arizona (United States)

Oleg G. Okhotnikov, Tampere University of Technology (Finland)

Edik U. Rafailov, University of Dundee (United Kingdom)

Alexei Sirbu, Ecole Polytechnique Fédérale de Lausanne (Switzerland)

Anne C. Tropper, University of Southampton (United Kingdom)

Session Chairs

1 CW VECSELs: Power Scaling I

Ursula Keller, ETH Zurich (Switzerland)

2 CW VECSELs: Power Scaling II

Oleg G. Okhotnikov, Tampere University of Technology (Finland)

3 Novel VECSEL Applications

Norman Hodgson, Coherent, Inc. (United States) 
$4 \quad$ Power Scaling VECSELs: Growth, Fabrication, and Electrical Pumping Bernd Witzigmann, Universität Kassel (Germany)

$5 \quad$ VECSELS: Theory and Simulations

Jerome V. Moloney, College of Optical Sciences, The University of Arizona (United States)

$6 \quad$ Ultrafast VECSELS and MIXSELS

Anne C. Tropper, University of Southampton (United Kingdom)

7 Ultrafast VECSELS

Uwe Griebner, Max-Born-Institut für Nichtlineare Optik und Kurzzeitspektroskopie (Germany)

8 Femtosecond VECSELs and Novel Applications

Thomas Südmeyer, ETH Zurich (Switzerland) 